\title{
Erratum
}

Obstetrics \&

\section{Correction of the name of author: Pre-implantation genetic diagnosis and pre-implantation genetic screening: two years experience at a single center}

Seyeon Won, Hannah Kim, Woo Sik Lee, Ji Won Kim, Sung Han Shim

In the published article, "Se Yeon Won, Hannah Kim, Woo Sik Lee, Ji Won Kim, and Sung Han Shim. Pre-implantation genetic diagnosis and pre-implantation genetic screening: two years experience at a single center. Obstet Gynecol Sci 2018;61(1):95101. https://doi.org/10.5468/ogs.2018.61.1.95," the name of author was published incorrectly. The authors apologize for any inconvenience that it may have caused.

Se Yeon Won, Hannah Kim, Woo Sik Lee, Ji Won Kim, Sung Han Shim

The name of author should be corrected as follows. Changes are marked by underlines:

Seyeon Won, Hannah Kim, Woo Sik Lee, Ji Won Kim, Sung Han Shim 